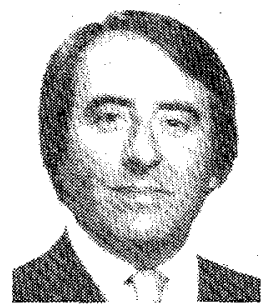

James Forrest Young (M'72) received the B.S. and M.S. degrees in electrical engineering from the Massachusetts Institute of Technology, Cambridge, in 1965 and 1966, and the Ph.D. degree in electrical engineering from Stanford University, Stanford, CA, in 1970.

Following his graduation, he joined Prof. S. E. Harris at Stanford University as a Research Associate with primary responsibility for the experimental activities of the group. In 1975 he was appointed Professor of Electrical Engineering (Research), and since 1977 he has also served as the Assistant Director for Technical Operations of the Edward L. Ginzton Laboratory. His areas of research have included optical parametric oscillators, nonlinear optical materials, nonlinear optical processes in vapors, infrared image upconversion, the development of UV and VUV radiation sources, laser induced inelastic collisions, and the development of experimental laser systems. He has served as a consultant to Coherent Radiation, Spectra-Physics, and Bell Telephone Laboratories.

Dr. Young is a Fellow of the Optical Society of America and a member of the American Physical Society, the Federation of American Scientists, Tau Beta $\mathrm{Pi}$, and Sigma Xi.

Stephen E. Harris (S'59-M'64-F'72) was born in Brooklyn, NY, in November 1936. He received the B.S. degree from Rennselaer Polytechnic Institute, Troy, NY, in 1959 and the M.S. and Ph.D. degrees from Stanford University, Stanford, CA, in 1961 and 1963, respectively, all in electrical engineering.

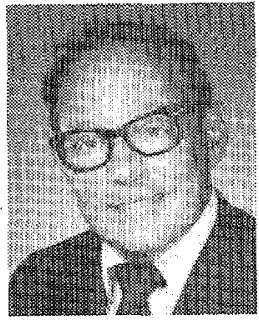

Since 1963 he has been on the faculty of Stanford University where he is now a Professor of Electrical Engineering and of Applied Physics. His research work has been in the fields of lasers, quantum electronics, nonlinear optics, acoustooptics, and atomic physics. Some of his research contributions include the invention of the FM laser, the first observation of optical parametric spontaneous emission, the operation of the first visible $\mathrm{CW}$ optical parametric oscillator, the invention of the tunable acoustooptic filter, the operation of the first electronically tunable laser, the invention and demonstration of generation of ultraviolet light by phasematched third harmonic generation in the metallic vapors and inert gases, and the use of this radiation to take the first hologram in the vacuum ultraviolet, the first observation of laser-induced inelastic collisions, and the invention of the tunable anti-Stokes XUV radiation source. His present interests are in the areas of xadiative collision lasers, and in the development of new techniques for generating extreme uItraviolet and soft X-ray radiation. He was one of the founders of Chromatix, Inc. in 1968, and has consulted for Sylvania Electronic Systems, Spectra-Physics, Westinghouse Electric Corporation, and for several government agencies. With the support of a Guggenheim Fellowship he spent the 1976-1977 academic year with the Department of Physics, Dartmouth College, Hanover, NH.

Dr. Harris is a Fellow of the Optical Society of America and the American Physical Society. In 1977 he was elected to the National Academy of Engineering, and in 1981 he was elected to the National Academy of Sciences. In past years he has received the 1965 Alfred Noble Prize of the American Society of Civil Engineers, the 1973 Curtis McGraw Research Award of the American Society for Engineering Education, and the 1978 David Sarnoff Award of the IEEE.

\title{
Emission of Short-Wavelength Photons from lon-Surface Charge Exchange
}

\author{
HAI-WOONG LEE AND THOMAS F. GEORGE
}

\begin{abstract}
The intensity of radiation emitted from ion-surface charge exchange processes can be significantly enhanced if the surface exposed to impinging ions is electronically excited. Alpha particles capturing electrons at a silicon surface are considered as possible candidates for a shiort-wavelength laser.
\end{abstract}

\section{INTRODUCTION}

I T has been proposed [1] that some selected charge exchange processes may serve as a means of achieving population inversion for short-wavelength lasers [2]. Recent observation [3] of strong VUV and X-ray emission from laser-produced

Manuscript received January 21, 1983; revised July 18, 1983. The work of H.-W. Lee was supported in part by the Petroleum Research Fund, administered by the American Chemical Society, a Grant from the Research Corporation and by an Oakland University Research Fellowship. The work of T. F. George was supported in part by the National Science Foundation under Grant CHE-8022874, the $f$ ir Force Office of Scientific Research (AFSC), United States Air Force, under Grant AFOSR-82-0046, the Office of Naval Research, and the U.S. Army Research Office.

H.-W. Lee is with the Department of Physics, Oakland University, Rochester, MI 48063.

T. F. George is with the Department of Chemistry, University of Rochester, Rochester, NY 14627. plasmas suggests a possibility of utilizing charge exchange in compound solids. For example, one might irradiate a compound solid with a high-power laser beam in order to preferentially excite one of the atomic species in the solid. The population inversion in the other atomic species could then be obtained through charge exchange that ensues between the two species.

Two independent investigations have focused on the possibility of using ion-surface charge exchange processes to obtain coherent short-wavelength radiation [4], [5] where the former [4] further suggested laser excitation of the surface. It is known that collisions of positive ions with a metal or semiconductor surface, e.g.,

$$
\mathrm{H}^{+}+\mathrm{Si}(111) \rightarrow \mathrm{H}+\mathrm{Si}^{+}(111)
$$

can produce excited atoms or ions and subsequent photon emission [6]. Under normal conditions, the intensity of emitted radiation is generally too low to even suggest the possibility for a laser. However, it has been suggested [4] that the intensity can be significantly enhanced if the surface exposed to impinging ions is electronically excited. Such excitation of a surface can be achieved by infrared or visible radiation 
which can effectively promote electrons to surface bands. As an illustration, we consider a collision of an alpha particle with an electronically excited silicon surface which produces excited singly ionized helium. Rough estimates of the charge exchange cross section and the laser gain are presented.

\section{THEORY}

When a positive ion $A^{m+}$ collides with a metal or semiconductor surface $S$, it can capture an electron at the surface.

$$
A^{m+}+S \rightarrow A^{(m-1)+}+S^{+} .
$$

The probability $P$ for this charge exchange to occur is given by [4]

$$
P=1-\exp \left[-2 \int_{-\infty}^{\infty} d t \Gamma(t)\right]
$$

where the instantaneous transition rate $\Gamma(t)$ can be written as

$$
\Gamma(t)=N_{E_{0}}^{2} \Gamma^{\prime}(t) \text {. }
$$

$\Gamma^{\prime}(t)$ is a complicated function which depends on various parameters of the ion and the solid, and $N_{E_{O}}$ is a normalization constant for an electron of energy $E_{O}$ in the solid where $E_{o}$ represents the resonance energy, i.e., the transfer of an electron of energy $E_{O}$ in the solid to the ion is an energy conserving process. The charge exchange cross section $\sigma$ can be estimated as

$$
\sigma \simeq \pi z_{o}^{2} P
$$

where $z_{o}$ is the ion-surface separation at which the electron capture occurs. We note that the normalization constant $N_{E_{0}}$ and consequently the charge exchange probability and cross section are generally much greater for a surface electron than for a bulk electron. This simply means that surface electrons are much easier to be picked up by the incoming ion than bulk electrons. The number density of the product $A^{(m-1)+}$ is therefore enhanced if bulk electrons are excited to surface bands. Such excitation of a surface can be achieved by irradiating the surface with a source of appropriate power and wavelength.

Regarding a possible short-wavelength laser, we of course are interested in the process in which the product $A^{(m-1)+}$ is produced predominantly in an excited state capable of emitting a short-wavelength photon, The gain of a Doppler-broadened medium is then given by the expression [7]

$$
g \cong \sqrt{\pi \ln 2} \frac{\lambda^{2}}{8 \pi} \frac{\Delta N}{\tau_{s} \Delta \nu}
$$

where $\Delta \nu$ and $\lambda$ are, respectively, the linewidth and wavelength of the transition in question, $\tau_{s}$ is the spontaneous lifetime of the upper level, and $\Delta N$ is the inversion density. In our case, $\Delta N$ is given by

$$
\Delta N \cong N_{\text {ion }} N_{e} v \sigma \tau_{s}
$$

where $N_{\text {ion }}$ and $N_{e}$ are the number densities of the incident ions and surface electrons, respectively (the effect of bulk electrons is neglected), and $v$ is the velocity of the incident ions. It is clear that in order to obtain the high gain required for laser action in the short-wavelength region, one needs a high inversion density $\Delta N$, which in turn puts the burden on the power of the excitation beam that must supply a sufficient number of surface electrons.

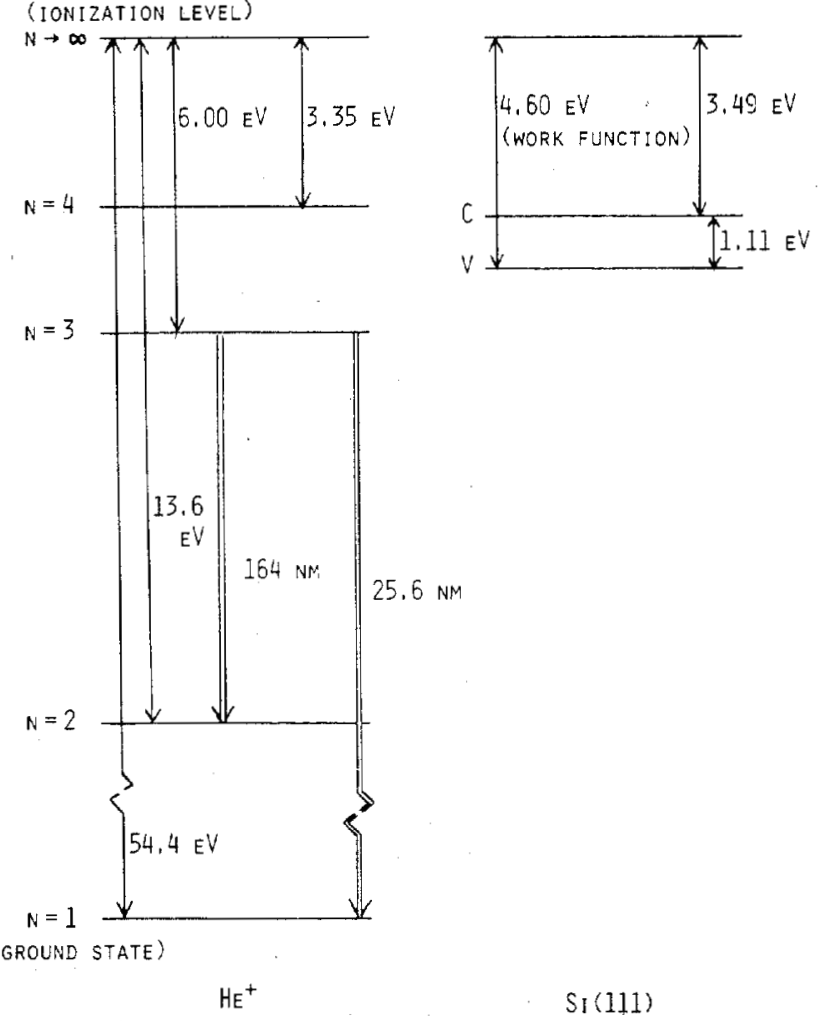

Fig. 1. Energy levels of $\mathrm{He}^{2+}+\mathrm{Si}(111)$. The valence and conduction bands are labeled by $V$ and $C$, respectively, with an energy gap of $1.11 \mathrm{eV}$.

\section{EXample-Alpha Particle at a SILICON SURFACE}

Let us consider an electron capture process of an alpha particle at a silicon(111) surface

$$
\mathrm{He}^{2+}+\mathrm{Si}(111) \rightarrow \mathrm{He}^{+}+\mathrm{Si}^{+} \text {. }
$$

A look at energy level structures of $\mathrm{He}^{+}$and a silicon surface (see Fig. 1) suggests that the helium ion is produced predominantly in its second excited state, $\mathrm{He}^{+}(3 l)$. Subsequent Balmer $\alpha$ and Lyman $\beta$ transitions produce radiation at $\lambda=164 \mathrm{~nm}$ and $\lambda=25.6 \mathrm{~nm}$, respectively.

The charge exchange probability $P$ as given by (1) can be written as

$$
P \approx 1-\exp \left[-\frac{4}{v} \int_{0}^{\infty} d z \Gamma(z)\right]
$$

where $z$ is the ion-surface separation, and a straight line trajectory with velocity $v$ is assumed for the ion. The evaluation of the above requires a detailed knowledge of the potential energy surfaces and surface states involved. Since the electron capture occurs at relatively large ion-surface separations, the potential energy surfaces are assumed to be determined mainly by image forces. For silicon, we adopt the one-dimensional model of a semiconductor [4] and take $W$ (work function) = $4.6 \mathrm{eV}, E_{g}$ (gap energy) $=1.11 \mathrm{eV}, \rho$ (density of surface states) $/$ area $=4 \times 10^{14} / \mathrm{eV} \cdot \mathrm{cm}^{2}$, and $K$ (dielectric constant $)=11.8$. The integral can then be carried out numerically, and we obtain $P \cong 0.097$ for the case $v=0.1$ a.u. $=2.2 \times 10^{7} \mathrm{~cm} / \mathrm{s}$. The resonance transfer of surface electrons occurs at $z_{0} \cong 7$ $12 a_{o}$ ( $a_{0}:$ Bohr radius), which yields $\sigma \cong 49 \pi \times 0.097 \cong$ $15.0 a_{o}^{2} \cong 4.19 \AA^{2}$ at $v=2.2 \times 10^{7} \mathrm{~cm} / \mathrm{s}$. This cross section appears to be sufficiently large to warrant our consideration of 
a short-wavelength laser utilizing the $\mathrm{He}^{2+}-\mathrm{Si}(111)$ system. However, one must supply a sufficiently high density of ions and, in particular, surface electrons in order to obtain the high gain. This problem is considered below.

For a short-wavelength laser, the gain $g$ is desired to be $\sim 10 / \mathrm{cm}$ or higher, requiring a high inversion density $\Delta N$ [see (4)]. For the Balmer $\alpha$ transition we have $\lambda=1.64 \times 10^{-5} \mathrm{~cm}$ and $\tau_{s}=1.4 \times 10^{-9}$ s. Taking $g=10 / \mathrm{cm}$ and the Doppler width $\Delta \nu \cong 10^{10} \mathrm{~Hz}$, we obtain $\Delta N \cong 8.9 \times 10^{12} / \mathrm{cm}^{3}$. With $v=2.2 \times 10^{7} \mathrm{~cm} / \mathrm{s}$ and $\sigma=4.19 \AA^{2}$ as calculated above and taking $N_{\text {ion }} \cong 10^{13} / \mathrm{cm}^{3}$, we then obtain, from (5), $N_{e} \cong$ $6.9 \times 10^{16} / \mathrm{cm}^{3}$. This corresponds to the surface electron density of $n_{e} \cong 7.6 \times 10^{9} / \mathrm{cm}^{2}$. The requirement on the power of the excitation source in order to populate this many surface electrons does not seem to be severe at all. We mention that a rough estimate shows that, with a power density of $1 \mathrm{~W} / \mathrm{cm}^{2}$ tuned at $\hbar \omega=2 E_{g} / 5$ ( $\omega=$ laser frequency), the number of electrons excited to surface bands is on the order of $10^{10} / \mathrm{cm}^{2} / \mathrm{s}$.

For the Lyman $\beta$ transition, we have $\lambda=2.56 \times 10^{-6} \mathrm{~cm}$ and $\tau_{s}=1.1 \times 10^{-9} \mathrm{~s}$. Taking again $g=10 / \mathrm{cm}$ and $\Delta \nu \cong 10^{10}$ $\mathrm{Hz}$, we obtain, from (4), $\Delta N \cong 2.9 \times 10^{14} / \mathrm{cm}^{3}$. Thus, the gain of $10 / \mathrm{cm}$ cannot be obtained unless $N_{\text {ion }} \geq 2.9 \times 10^{14} /$ $\mathrm{cm}^{3}$. If we take $N_{\text {ion }}=10^{14} / \mathrm{cm}^{3}$ and accordingly $\Delta N=10^{14} /$ $\mathrm{cm}^{3}$, we obtain, using (4), the maximum possible gain of $g \cong 3.5 / \mathrm{cm}$. In order to achieve $\Delta N \cong 10^{14} / \mathrm{cm}^{3}$, one must have $N_{e}=9.9 \times 10^{16} / \mathrm{cm}^{3}$ [see (5)] which corresponds to the surface density $n_{e} \cong 1.1 \times 10^{10} / \mathrm{cm}^{2}$. The requirement on the power of the source to excite this many electrons is again not severe.

It should be noted that in our treatment we have neglected Auger processes which may contribute to the decrease of gain. Through Auger processes, incident alpha particles can produce helium ions in their ground state accompanied by ejection of electrons [8], [9]. Furthermore, excited helium ions produced by charge exchange may be quenched by Auger deexcitation before they escape the surface [8], [9]. Although there are some indications [8] that the probability for Auger deexcitation is small for the velocity range $\left(\sim 10^{7} \mathrm{~cm} / \mathrm{s}\right)$ considered here, it seems desirable that the relative importance of resonance charge exchange compared to Auger processes (especially for surface electrons) be further investigated. We also mention that our analysis is based on the one-dimensional model [4] of a surface according to which a semiconductor has a direct gap. In reality, however, semiconductors like silicon have an indirect gap, and the excitation of electrons into surface bands by radiation may have to be accompanied by phonon excitations $[10]$.

\section{ACKNOWLEDGMENT}

T. F. George acknowledges the Camile and Henry Dreyfus Foundation for a Teacher-Scholar Award (1975-1984) and the John Simon Guggenheim Memorial Foundation for a Fellowship (1983-1984).

\section{REFERENCES}

[1] A. V. Vinogradov and I. I. Sobel'man, "The problem of laser radiation sources in the far ultraviolet and X-ray regions," Sov. Phys. JETP, vol. 36, pp. 1115-1119, 1973; M. O. Scully, W. H. Louisell, and W. B. McKnight, "A soft X-ray laser utilizing charge exchange," Opt. Commun., vol. 9, pp. 246-248, 1973; W. H.
Louisell, M. O. Scully, and W. B. McKnight, "Analysis of a soft X-ray laser with charge-exchange excitation," Phys. Rev. A, vol. 11, pp.989-1000, 1975; D. Anderson, J. McCullen, M. O. Scully, and J. F. Seely, "Analysis of short-wavelength charge-exchange lasers via plasma-gas beam clashing," Opt. Commun., vol. 17, pp. 226-230, 1976; D. A. Copeland, H. Mahr, and C. L. Tang, "Threshold and rate equation considerations for a $\mathrm{H}^{+}$-Cs chargeexchange laser," IEEE J. Quantum Electron. vol. QE-12, pp. $665-673,1976$

[2] M. A. Duguay, "X-ray lasers: A status report," Laser Focus, vol. 9, pp. 41-46, 1973; H. Mahr and U. Roeder, "Use of metastable ions for a soft X-ray laser," Opt. Commun., vol. 10, pp. 227 228, 1974; B. Lax, A. H. Guenther, D. R. Cohn, and W. Halverson, "Feasibility of X-ray lasers," in Laser Interaction and Related Plasma Phenomena, vol. 3B. H. J. Schwarz and H. Hora, Eds. New York: Plenum, 1974, pp. 859-874; A. Ferguson, "How to make an x-ray laser," New Scientist, vol. 68, pp. 207209, 1975; G. Chapline and L. Wood, "X-ray lasers," Phys. Today, vol. 28 , pp. $40-48,1975$; S. A. Mani, H. A. Hyman, and J. D. Dougherty, "Lithium-ion soft X-ray laser," J. Appl. Phys., vol. 47, pp. 3099-3106, 1976; R. W. Waynant and R. C. Elton, "Review of short-wavelength laser research," Proc. IEEE, vol. 64, pp. 1059-1092, 1976; G. J. Pert, "X-ray lasers," in Laser Advances and Applications, Proc. 4th Nat. Quantum Electron. Conf. Edinburgh, New York: Wiley, 1979, pp. 69-75; D. Jacoby, G. J. Pert, S. A. Ramsdem, L. D. Shorrock, and G. J. Tallents, "Observation of gain in a possible extreme ultraviolet lasing system," Opt. Commun., vol. 37, pp. 193-196, 1981; I. I. Sobel'man, "Atomic collision processes and uv and X-ray lasers," in Electronic and Atomic Collisions, N. Oda and K. Takayanagi, Eds. Amsterdam: North-Holland, 1980, pp. 75-80; V. A. Bhagavatula, "Soft $\mathrm{X}$-ray population inversion in laser plasmas by resonant photoexcitation and photon-assisted processes," IEEE J. Quantum Electron., vol. QE-16, pp. 603-618, 1980; J. E. Rothenberg and S. E. Harris, "XUV lasers by quartet to doublet energy transfer in alkali atoms," IEEE J. Quantum Electron., vol. QE-17, pp. 418422, 1981; C. A. Nicolaides and Y. Komminos, "Possibility for VUV and X-ray tunable atomic lasers," Chem. Phys. Lett., vol. 80 , pp. 463-468, 1981.

[3] R. H. Dixon and R. C. Elton, "Resonant charge transfer and population inversion following $\mathrm{C}^{5+}$ and $\mathrm{C}^{6+}$ interactions with carbon atoms in a laser-generated plasma," Phys. Rev. Lett., vol. 38, pp. 1072-1075, 1977; B. Yaakobi, P. Bourke, Y. Conturie, J. Delettrez, J. M. Forsyth, R. D. Frankel, L. M. Goldman, R. L. McCroy, W. Seka, J. M. Soures, A. J. Burek, and R. E. Deslattes, "High X-ray conversion efficiency with target irradiation by a frequency tripled Nd:glass laser," Opt. Commun., vol. 38, pp. 196-200, 1981; R. L. Carman and G. F. Chapline, "Suprathermal electron pumping of X-ray lasers," in Proc. Int. Conf. Lasers ' 81 , C. B. Collins, Ed., McLean, VA: STS, 1981, pp. 173-176; G. J. Pert, "Observation of gain in the XUV spectral region," in Proc. Int. Conf. Lasers '81, C. B. Collins, Ed., McLean, VA: STS, 1981, p. 177; G. Jamelot, P. Jaeglé, A. Carrillon, A. Bideau, C. Müller, H. Guennou, and A. Sureau, "Evidence of population inversion in Li-like aluminum ions in a laser produced plasma," in Proc. Int. Conf. Lasers ' 81 , C. B. Collins, Ed., McLean, VA: STS, 1981, pp. 178-183.

[4] H. W. Lee, W. C. Murphy, and T. F. George, "Neutralization of ions at an electronically excited semiconductor surface," Chem. Phys, Lett., vol. 93, pp. 221-225, 1982.

[5] J. S. Helman, C. Rau, and C. F. Bunge, "X-ray laser implementation by means of a strong source of high-spin metastable atoms," Phys. Rev. A, vol. 27, pp. 562-564, 1983.

[6] E. O. Rausch and E. W. Thomas, "Formation of excited $\mathrm{H}$ by impact of $5-30 \mathrm{keV} \mathrm{H} \mathrm{H}_{1}^{+}, \mathrm{H}_{2}^{+}$, and $\mathrm{H}_{3}^{+}$ions on metal surfaces," Phys. Rev. A, vol. 14, pp. 1912-1914, 1976; S. Y. Leung, N. J. Tolk, W. Heiland, J. C. Tully, J. S. Kraus, and P. Hill, "Optical radiation from low-energy hydrogen atomic and molecular ionsurface collisions," Phys. Rev. A, vol. 18, pp. 447-451, 1978; C. W. White, E. W. Thomas, W. F. van der Weg, and N. H. Tolk, "Optical emission from low-energy ion-surface collisions," in Inelastic Ion-Surface Collisions, N. H. Tolk, J.C. Tully, W. Heiland, and C. W. White, Eds. New York: Academic, 1977, pp. 201-252.

[7] A. Yariv, Quantum Electronics, 2nd Ed. New York: Wiley, 1975 , ch. 8 .

[8] A. Cobas and W. E. Lamb, "On the extraction of electrons from a metal surface by ions and metastable atoms," Phys. Rev., vol. 65, pp. 327-337, 1944

[9] H. D. Hagstrum, "Theory of Auger ejection of electrons from metals by ions," Phys. Rev., vol, 96, pp. $336-365,1954 ;-$ 
"Theory of Auger neutralization of ions at the surface of a diamond-type semiconductor," Phys. Rev., vol. 122, pp. 83-113, $1961 ;$ - "Excited-atom deexcitation spectroscopy using incident ions," Phys. Rev. Lett., vol. 43, pp. 1050-1053, 1979.

[10] W. C. Murphy, A. C. Beri, T. F. George, and J. Lin, "Analysis of laser-enhanced adsorption/desorption processes on semiconductor surfaces via electronic surface state excitation," in Proc. Mat. Res. Soc. Symp., vol. 17, pp. 273-282, 1983.

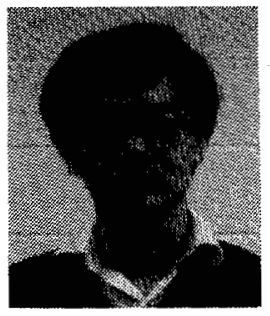

Hai-Woong Lee was born in Seoul, Korea, in 1947. He received the B.S. degree from Seoul National University in 1970 and the Ph.D. degree from the University of Pittsburgh, Pittsburgh, PA, in 1977.

After doing postdoctoral work at the University of Rochester, Rochester, NY, the University of Arizona, Tucson, and the University of New Mexico, Albuquerque, he joined the faculty of Oakland University, Rochester, MI, in 1981 where he is an Assistant Professor of Physics. His research activities include theoretical quantum optics and collision theory.
Dr. Lee is a member of the American Physical Society, the Optical Society of America, and Sigma Xi.

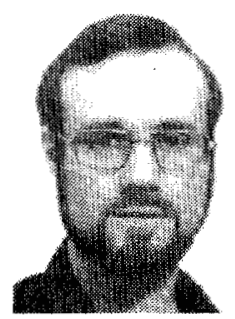

Thomas F. George was born in Philadelphia, PA, on March 18, 1947. He received the B.A. degree in chemistry and mathematics from Gettysburg College, Gettysburg, PA, in 1967, and the M.S. and Ph.D. degrees in chemistry from Yale University, New Haven, CT, in 1968 and 1970 , respectively.

During 1970-1971 he was a Research Associate at the Massachusetts Institute of Technology, Cambridge, and during 1971-1972 he was a Postdoctoral Appointee at the University of California, Berkeley. In September 1972 he joined the staff of the University of Rochester, Rochester, NY, as Assistant Professor of Chemistry. He became an Associate Professor in 1974 and a Professor in 1977. His research interests include molecular energy transfer, reaction dynamics, and the interaction of laser radiation with molecular rate processes in the gas phase and at a solid surface.

Dr. George is a member of the American Chemical Society, the American Physical Society, the Royal Society of Chemistry, the Society of Photo-Optical Instrumentation, and is a Fellow of the New York Academy of Sciences. He is on the Advisory Board of the Journal of Physical Chemistry:

\title{
Table-Top EUV Continuum Light Source
}

\author{
P. K. CARROLL, EUGENE T. KENNEDY, AND GERARD O'SULLIVAN
}

\begin{abstract}
Work in recent years on the development of a convenient "table-top" source of continuum radiation in the XUV and VUV from 4 to $200 \mathrm{~nm}$ is summarized. It was found that laser-produced plasmas on targets of high atomic number $(62 \leqslant Z \leqslant 74)$ emitted apparently line-free continua over very substantial spectral regions in the XUV and VUV. The continua are very intense, reproducible, and relatively insensitive to ambient pressure. The effective absence of line emission can be explained on the basis of the electron configurations of the ionized species responsible for the emission. A high repetition rate modular version of the source is described. Applications and advantages of the light source are presented.
\end{abstract}

\section{INTRODUCTION}

$\mathrm{T}$ HE development of the synchrotron as a source of continuum radiation has led, in recent years, to a considerable growth of activity in such areas as absorption spectroscopy and photoionization in the VUV and the XUV regions. Although very effective as a light source, the synchrotron has the extreme disadvantages of being very costly and nonportable, and the need for a much simpler and cheaper light source is very apparent. We believe that the simple compact light

Manuscript received February 7, 1983; revised September 2, 1983. This work was supported in part by the National Board for Science and Technology (Ireland) under Research Grant URG/143/82 and by the NSF under Grant PHT 80-16657.

P. K. Carroll is with the Department of Physics, University College, Dublin, Ireland.

E. T. Kennedy and G. O'Sullivan are with the School of Physical Sciences, National Institute for Higher Education, Dublin, Ireland. source described in the present paper and which was developed initially in the Department of Physics, University College, Dublin, Ireland, has much to offer and indeed has been used successfully on a routine basis as a table-top source in our laboratory $[1],[2]$.

\section{EARLY EXPERIMENTS WITH Laser-Produced Continua}

Shortly after the development of the giant pulse $Q$-switch laser it was found that when its output was focused on a solid target material in vacuum, a dense high-temperature plasma was generated. The spectra of such plasmas were found typically to originate in species in high stages of ionization. Many new spectra were generated and studied in this way; as typical examples, we quote the work of Fawcett [3] and Doschek et al. [4]. In all these cases the spectra were dominated by strong line emission and the thrust of the work was on conventional term analysis. To a greater or lesser extent continuum originating in recombination and bremsstrahlung was always present. Because of the dominance of strong lines, however, the continuum was quite unsuitable as a background for absorption studies.

A systematic study of target materials was undertaken in this laboratory with the hope of finding a spectrum in which the lines would be, to a greater or lesser degree, suppressed with respect to the continuum. In the course of this work almost all the heavier elements from strontium $(Z=38)$ to uranium 